\title{
A DFT Investigation of Triarylamine-a-Cyanoacrylic Acid Compounds: Structural, Electronic, And Nonlinear-Optical Properties
}

\section{Ziran Chen}

Sichuan Vocational and Technical College

\section{Yuhong Zhang}

Sichuan Vocational and Technical College

\section{Yuan Li}

Sichuan Vocational and Technical College

Wenhao Yu ( $\square$ yuwenhao@sicnu.edu.cn )

Sichuan Normal University https://orcid.org/0000-0003-2991-5362

\section{Research Article}

Keywords: Triarylamine-a-cyanoacrylic acid囚Density functional theory, Finite field method, Nonlinearoptical properties, Molecular modeling

Posted Date: February 7th, 2022

DOI: https://doi.org/10.21203/rs.3.rs-1304695/v1

License: (9) This work is licensed under a Creative Commons Attribution 4.0 International License. Read Full License 


\title{
A DFT Investigation of Triarylamine-a-cyanoacrylic Acid Compounds: Structural, Electronic, and Nonlinear-Optical Properties
}

\author{
Ziran Chen ${ }^{\mathrm{a}}$, Yuhong Zhang ${ }^{\mathrm{a}}$, Yuan $\mathrm{Li}^{\mathrm{a},{ }^{*}}$, and Wenhao $\mathrm{Yu}^{\mathrm{b}, \mathrm{c}^{*}}$ \\ ${ }^{a}$ Department of Architecture and Environment Engineering, Sichuan Vocational and Technical \\ College, Suining, Sichuan 629000, P. R. China \\ ${ }^{b}$ College of Chemistry and Material Science, Sichuan Normal University, Chengdu, Sichuan 610068, \\ P. R. China \\ ${ }^{\mathrm{c}}$ College of Polymer Science \& Engineering, State Key Laboratory of Polymer Materials \\ Engineering, Sichuan University, Chengdu, 610065, P. R. China. \\ Corresponding author: \\ Wenhao Yu *Email: yuwenhao@sicnu.edu.cn
}

YUAN LI *Email: 30278300@qq.com

Abstract: Using density functional theory and finite field method, nonlinear-optical properties of nine triarylamine- $\alpha$-cyanocinnamic acid molecules was calculated at the theoretical level of M06-2X/6-311++G(d,p) and WB97XD/6-311++G(d,p). Except for (E)-2-cyano-3-(4-(di([1,1'-biphenyl]-4-yl)amino)phenyl)acrylic acid (a) which is D- $\pi$-A electronic structure, the remaining eight derivative molecules are all A- $\pi$-D- $\pi$-A structures. The calculation result showed that the lowest energy transition of the nine triarylamine derivatives molecules was the $\pi-\pi^{*}$ electron transition from HOMO to LUMO. Compared with those of the gas phase, the maximum absorption wavelengths of the molecules in ethanol solution exhibited a certain degree of redshift. The introduction of conjugated carbon-carbon double bonds or carbon-carbon triple bonds between the biphenyl structure of molecule a has little effect on the second-order nonlinear-optical properties of the molecules. However, the introduction of carbon-carbon double bonds into the parent molecules improves the third-order nonlinear-optical properties. The introduction of a heterocyclic ring (furan ring or thiophene ring) between the cyano-cinnamic acid group branch and the triarylamine enhances the second-order and third-order nonlinear-optical properties, especially the second-order and third-order polarisability of the $\mathbf{b 3}$ and $\mathbf{c 3}$ molecules obtained by introducing a thiophene ring; these molecules had the highest polarisability values. The second- and third-order polarizabilities of the $\mathbf{b 3}$ molecule are $0.13 \times 10^{5}$ a.u. and $27.13 \times 10^{5}$ a.u., respectively, and the second- 
and third-order polarizabilities of the $\mathbf{c} 3$ molecule are $0.14 \times 10^{5}$ a.u. and $28.10 \times 10^{5}$ a.u., respectively. This suggests that the $\mathbf{b} \mathbf{3}$ and $\mathbf{c} 3$ molecules have beneficial second- and third-order nonlinear-optical properties, and can be used as the basis for the design of good second- and third-order nonlinear-optical materials.

Keywords: Triarylamine- $\alpha$-cyanoacrylic acid, Density functional theory, Finite field method, Nonlinear-optical properties, Molecular modeling 


\section{Introduction}

Compared with traditional inorganic nonlinear-optical materials, organic nonlinear-optical materials have excellent characteristics such as high electro-optic coefficients, fast response speeds, and high chemical and thermal stability. ${ }^{1,2}$ Their molecular structures are readily modified, and they are also easy to process. Furthermore, they have a broad range of potential applications in the fields of high-speed optical communication, optical information processing, optical storage, and high-frequency (terahertz) electro-optic devices, among others. The design of organic nonlinear-optical molecules with high nonlinear-optical coefficients is of great interest to theoretical and experimental researchers ${ }^{3-7}$. Although a large number of nonlinear-optical chromophores have been developed in the past two decades, only a few of them have shown good performance in optoelectronic devices ${ }^{8},{ }^{9}$. Therefore, further enhancement of their nonlinear-optical properties requires rational molecular-structure design based on a deep understanding of structure-property relationships. Density-functional theory (DFT) and time-dependent density-functional theory (TD-DFT) are widely used in the computational research of organic nonlinear-optical properties ${ }^{3-5}$, 10-16.

High-performance nonlinear-optical materials can be designed with novel electron-acceptor (A) and electron-donor (D) structures by ordering the connections of A, D, and $\pi$-conjugated systems $(\pi)$; for example, $D-\pi-A, A-\pi-D-\pi-A$, or D- $\pi-A-\pi-D$; or by changing the substituents on $A, \pi$, and $D^{6,17}$. In the reported optical materials of the triarylamine- $\alpha$-cyanocinnamic acid system, triphenylamine (TPA) is typically used as an electron donor and cyanocinnamic acid is used as an electron acceptor, and these two parts are bridged by the $\pi$-conjugated system to form organic dyes ${ }^{18-21}$. TPA, as the electron donor, transfers the LUMO+1 orbital of the organic system to a lower energy level, which lowers the energy gap between LUMO and LUMO+1 and results in a wider absorption spectrum ${ }^{18}$. Because the acceptor and donor are connected by the $\pi$-conjugated system, intramolecular charge transfer from the donor to the acceptor pre-polarises the electron ground state ${ }^{22,23}$. For example, Janjua et al. ${ }^{24}$ used TD-DFT to predict the second-order nonlinear optical properties of triphenylamine- $\alpha$-cyanocinnamic acid derivatives conjugated with acetylenyl benzene. The static second-order polarisability $\left(\beta_{\text {tot }}\right)$ showed that the intramolecular charge transfer along the triphenylamine group to $\alpha$-cyanocinnamic acid played a key role in the nonlinear-optical response, and the addition of electron-acceptor fluorine atoms in the phenyl-conjugated system increased the $\beta$ value. 
In this study, the Gaussian 16 A.03 program ${ }^{25}$ was used to optimise the molecular structure of nine triarylamine- $\alpha$-cyanocinnamic acid molecules, as shown in Figure 1. Theoretical electronic spectra and nonlinear-optical properties were also computed. The influences of changes in electron-donor structure and the introduction of $\pi$-conjugated heterocyclic rings on nonlinear-optical properties were systematically investigated. Specifically, based on the structure of the parent molecule a, (E)-2-cyano-3-(4-(di([1,1'-biphenyl]-4-yl)amino)phenyl)acrylic acid, unsaturated bonds (carbon-carbon double bonds and carbon-carbon triple bonds) were added to expand the conjugated system. Furthermore, $\pi$-conjugated heterocycles (furan rings and thiophene rings) were introduced into the D-A triarylamine- $\alpha$-cyanocinnamic acid system to form a D- $\pi$-A structure. The results provided a theoretical framework for further design and synthesis of triarylamine- $\alpha$-cyanocinnamic acid compounds with excellent nonlinear-optical properties.

Figure 1. The chemical structures of nine triarylamine- $\alpha$-cyanoacrylic acid compounds.

\section{Computational details}

The experiment usually uses the electric field induced second harmonic generation method to measure the second-order NLO properties $\beta_{\mu}$. $\beta_{\mu}$ is the projection of $\beta$ in the direction of the dipole moment. In this article, in order to provide reference data for the experiment, the formulas used are as follows $4,5,13,14$ :

$$
\begin{aligned}
& \beta_{\mu}=\left(\mu_{\mathrm{x}} \beta_{\mathrm{x}}+\mu_{\mathrm{y}} \beta_{\mathrm{y}}+\mu_{\mathrm{z}} \beta_{\mathrm{z}}\right) /\left(\mu_{\mathrm{x}}^{2}+\mu_{\mathrm{y}}^{2}+\mu_{\mathrm{z}}^{2}\right)^{1 / 2} \\
& \beta_{i}=1 / 3 \sum_{k}\left(\beta_{i k k}+\beta_{k i k}+\beta_{k k i}\right) \quad i, k=\mathrm{x}, \mathrm{y}, \mathrm{z} \\
& \beta_{0}=\left(\beta_{\mathrm{x}}^{2}+\beta_{\mathrm{y}}^{2}+\beta_{\mathrm{z}}^{2}\right)^{1 / 2}
\end{aligned}
$$

where $\mu_{\mathrm{x}}, \mu_{\mathrm{y}}, \mu_{\mathrm{z}}$ are the components of the dipole moment in the $\mathrm{x}, \mathrm{y}$, and $\mathrm{z}$ direction, respectively. $\beta_{i}$ is the component of second-order nonlinear optical property in direction $i$, and $\beta_{i k k}$ is the third-order tensor component. The finite field method is an effective method for calculating the NLO properties of organic molecules $\mathbf{4 , 2 6}^{2}$. In this paper, using the finite field method, the hybrid functional M06-2X and the HF long-range correction hybrid functional WBXD 27-30 mixed with $100 \%$ in the progressive region, calculate the third-order tensor components of its 
second-order NLO properties respectively. The hybrid functional M06-2X is used to calculate the fourth-order tensor components of its third-order NLO properties, and the values of $\beta_{\mu}, \beta_{0}$ and $\gamma$ are obtained by formulas (1), (3) and (4).

$\gamma=\left(\gamma_{x x x x}+\gamma_{y y y y}+\gamma_{z z z z}+2 \gamma_{x x y y}+2 \gamma_{x x z z}+2 \gamma_{y y z z}\right) / 5$

where the $\gamma_{\mathrm{xxxx}}, \gamma_{\mathrm{yyyy}}, \gamma_{\mathrm{zzzz}}, \gamma_{\mathrm{xxyy}}, \gamma_{\mathrm{xxzz}}, \gamma_{\mathrm{yyzz}}$ are the fourth-order tensor components. The center of $\mathrm{N}$ atom of triarylamine is chosen as the coordinate origin when calculating the NLO properties. The the $\mathrm{X}$ axis is located on the benzene ring containing the cyanocinnamic acid branch, the $\mathrm{Z}$ axis is perpendicular to the conjugate plane formed by the $\mathrm{N}$ atom and the benzene ring, and the dipole moment of the molecule is roughly the same as the $\mathrm{X}$ axis.

\section{Results and discussion}

\subsection{Geometric structures}

Calculations were optimised at the M06-2X /6-311++G(d,p) level to obtain stable configurations of the triarylamine derivative molecules, without imaginary frequencies, as shown in Figure S1. The dihedral angle values of the two benzene rings in the molecule obtained by structural optimisation are shown in Table S1. According to this data, the two benzene rings in the a- and c-series molecular structures were not coplanar, while those in the $\mathbf{b}$-series molecular structures were almost coplanar.

Bond order is a numerical value indicating the bonding strength between two adjacent atoms in a molecule. When the bond-order value is close to 1 , a covalent single bond is formed between the two atoms. When the value is close to 2 , covalent double bonds are formed; when it is between 1 and 1.5, a conjugate bond intermediate between a single bond and double bond forms. The Wiberg bond-order values calculated by the M06-2X method are shown in Figure S2. It can be seen for the nine molecular structures studied that the values for all carbon-carbon single bonds, carbon-oxygen single bonds, and carbon-sulphur single bonds were between 1 and 1.3. This indicates good molecule conjugation, which is beneficial to charge flow within each molecule.

\subsection{Electronic structures}

On this basis, the TD-M06-2X method and the same basis set were used to calculate the excited-state properties of the molecules in the gas phase and ethanol solvent, respectively, and the 
frontier molecular orbital and electronic absorption spectra data were obtained (Figure $\mathbf{2}$ and Table 1, respectively). The energy-gap values of molecules $\mathbf{a}, \mathbf{b}$, and $\mathbf{c}$, and the electron-cloud distributions of the frontier molecular orbitals (HOMO and LUMO), were close to the 3.06, 2.83, and $2.90 \mathrm{ev,}$ respectively, reported in the literature ${ }^{\mathbf{1 0}}$. By analysing the data in Figure 1, it can be seen that the energy-gap values of molecules decreased in the order $\mathbf{a}, \mathbf{b}, \mathbf{c} ; \mathbf{b}, \mathbf{b 1}, \mathbf{b 2}, \mathbf{b 3}$; and $\mathbf{c}, \mathbf{c 1}, \mathbf{c} 2$, $\mathbf{c} 3$, which indicates that the introduction of conjugated carbon-carbon double bonds and carbon-carbon triple bonds into parent molecule a, and the introduction of a benzene ring, furan ring or thiophene ring between the branched-chain containing cyanocinnamic acid group and triarylamine were all conducive to electronic transitions within the molecule. The HOMO electron clouds (which are bond-making $\pi$ orbitals) of each of the nine studied molecules are uniformly distributed over the entire molecule, while the LUMO electron clouds (which are anti-bonding $\pi^{*}$ orbitals) are all distributed on the branched-chain-containing cyanocinnamic acid group.

The maximum ultraviolet-absorption wavelengths of molecules $\mathbf{a}, \mathbf{b}$, and $\mathbf{c}$ in Table $\mathbf{1}$ were close to the values 410,410 , and $424 \mathrm{~nm}$, respectively, reported in the literature ${ }^{\mathbf{1 0}}$. The electronic transitions of the nine molecules in low-energy excited states all occurred between HOMO and LUMO, mainly due to the $\mathrm{S}_{0} \rightarrow \mathrm{S}_{1}$ transition. The maximum absorption wavelengths of the molecules were in the range of 388-434 nm, which is in the visible light range. From the data in Table 2, it can be seen that the absorption spectra of the studied molecules in ethanol solution was redshifted by 6-31 nm, compared with those in a gas phase environment.

\subsection{NPA charge analysis}

In order to explore the correlation between charge transfer and nonlinear-optical properties of organic nonlinear-optical materials, the charge distribution of the nine studied molecules was calculated by the natural bond orbital (NBO) method. The natural population analysis (NPA) charge values of each part of the molecules are listed in Table 2, where I is two benzene rings at the ends of two biphenyl-containing branched chains in triarylamine derivatives, II is an ethynyl-conjugated bridge or vinyl-conjugated bridge between two biphenyls in triarylamine derivatives, III is benzene rings, furan rings, and thiophene rings introduced from triarylamine or triarylamine and cyanocinnamic acid-containing branched chains, and IV is cyanocinnamic acid group.

Figure 2. HOMO energies $\left(E_{H}\right)$, LUMO energies $\left(E_{L}\right)$, and HOMO-LUMO gaps $\left(E_{g}\right)$ of nine triarylamine- $\alpha$-cyanoacrylic acid compounds. 
Table 1. The electronic absorption spectra of nine triarylamine- $\alpha$-cyanoacrylic acid compounds at the M06-2X/6-311++G(d,p) theoretical level.

\begin{tabular}{|c|c|c|c|c|c|}
\hline & Comp. & $\lambda / \mathrm{nm}$ & $E_{\mathrm{g}} / \mathrm{ev}$ & $F$ & Major contribution \\
\hline \multirow[t]{9}{*}{ Gas phase } & $\mathbf{a}$ & 378 & 3.28 & 1.0203 & HOMO $\rightarrow$ LUMO $(91 \%)$ \\
\hline & b & 387 & 3.20 & 1.1014 & $\mathrm{HOMO} \rightarrow \mathrm{LUMO}(87 \%)$ \\
\hline & c & 393 & 3.15 & 1.0158 & $\mathrm{HOMO} \rightarrow$ LUMO $(87 \%)$ \\
\hline & b1 & 382 & 3.25 & 1.0697 & $\mathrm{HOMO} \rightarrow \mathrm{LUMO}(77 \%)$ \\
\hline & b2 & 407 & 3.04 & 1.4551 & $\mathrm{HOMO} \rightarrow \mathrm{LUMO}(76 \%)$ \\
\hline & b3 & 404 & 3.07 & 1.3352 & $\mathrm{HOMO} \rightarrow \mathrm{LUMO}(75 \%)$ \\
\hline & c1 & 392 & 3.16 & 0.9814 & $\mathrm{HOMO} \rightarrow \mathrm{LUMO}(78 \%)$ \\
\hline & c2 & 415 & 2.98 & 1.3447 & $\mathrm{HOMO} \rightarrow \mathrm{LUMO}(76 \%)$ \\
\hline & c3 & 414 & 3.00 & 1.2350 & $\mathrm{HOMO} \rightarrow$ LUMO $(76 \%)$ \\
\hline \multirow{9}{*}{$\begin{array}{l}\text { Ethanol } \\
\text { solvent } \\
\text { system }\end{array}$} & $\mathbf{a}$ & $409\left(410^{*}\right)$ & 3.03 & 1.1836 & $\mathrm{HOMO} \rightarrow$ LUMO (91\%) \\
\hline & b & $405\left(410^{*}\right)$ & 3.05 & 1.2234 & $\mathrm{HOMO} \rightarrow$ LUMO $(87 \%)$ \\
\hline & c & $416\left(424^{*}\right)$ & 2.98 & 1.1194 & $\mathrm{HOMO} \rightarrow$ LUMO $(87 \%)$ \\
\hline & b1 & 388 & 3.19 & 1.1331 & $\mathrm{HOMO} \rightarrow \mathrm{LUMO}(77 \%)$ \\
\hline & b2 & 427 & 2.90 & 1.5425 & $\mathrm{HOMO} \rightarrow \mathrm{LUMO}(76 \%)$ \\
\hline & b3 & 420 & 2.95 & 1.4491 & $\mathrm{HOMO} \rightarrow \mathrm{LUMO}(75 \%)$ \\
\hline & c1 & 403 & 3.08 & 1.0914 & $\mathrm{HOMO} \rightarrow \mathrm{LUMO}(78 \%)$ \\
\hline & c2 & 415 & 2.98 & 1.3447 & $\mathrm{HOMO} \rightarrow \mathrm{LUMO}(76 \%)$ \\
\hline & c3 & 434 & 2.86 & 1.3164 & HOMO $\rightarrow$ LUMO $(76 \%)$ \\
\hline
\end{tabular}

Electronic transition: $\mathrm{S}_{0} \rightarrow \mathrm{S}_{1}$. The data in brackets are experimental values in ethanol solution. ${ }^{\mathbf{3 1}}$

Table 2. NPA charge distribution of nine triarylamine- $\alpha$-cyanoacrylic acid compounds

\begin{tabular}{cccccccccc}
\hline Comp. & $\mathbf{a}$ & $\mathbf{b}$ & $\mathbf{c}$ & $\mathbf{b 1}$ & $\mathbf{b 2}$ & $\mathbf{b 3}$ & $\mathbf{c 1}$ & $\mathbf{c 2}$ & $\mathbf{c 3}$ \\
\hline $\mathrm{Q}_{\mathrm{I}} / \mathrm{e}$ & 0.031 & -0.019 & -0.048 & -0.030 & -0.027 & -0.027 & -0.060 & -0.057 & -0.057 \\
$\mathrm{Q}_{\mathrm{II}} / \mathrm{e}$ & & 0.107 & 0.118 & 0.100 & 0.101 & 0.101 & 0.112 & 0.112 & 0.111 \\
$\mathrm{Q}_{\mathrm{III}} / \mathrm{e}$ & 0.103 & 0.024 & 0.062 & 0.018 & 0.077 & 0.054 & 0.029 & 0.089 & 0.073 \\
$\mathrm{Q}_{\mathrm{IV}} / \mathrm{e}$ & -0.115 & -0.103 & -0.102 & -0.079 & -0.139 & -0.119 & -0.081 & -0.143 & -0.052 \\
\hline
\end{tabular}

It can be seen from Table 2 that structural units II and III of the nine molecules were positively charged, and played the role of D in the molecules. Except for the parent molecule a, the I and IV structural units were negatively charged, and played the role of $\mathrm{A}$ in the molecules. Therefore, molecule a can be regarded as a D- $\pi$-A structure, and the other eight molecules can be regarded as A- $\pi$-D- $\pi$-A structures. Therefore, the introduction of carbon-carbon double bonds and carbon-carbon 
triple bonds between biphenyls in molecule a, as well as the introduction of a benzene ring, furan ring, and thiophene ring in the branched-chain-containing cyanocinnamic acid group changed the charge distribution of the molecules. With the introduction of a heterocyclic ring into $\mathbf{b}$-series and c-series derivatives, with $\mathbf{b}$ and $\mathbf{c}$ as parents, the charge value of structural unit II was almost unchanged, while the charge values of the other structural units were significantly increased, indicating that the delocalisation of electrons was enhanced.

\subsection{Nonlinear-optical response properties of the molecules}

\subsubsection{Second-order nonlinear-optical properties}

For the nine optimised, stable triarylamine structures, the hybrid functional M06-2X and the long-range-corrected hybrid functional WB97XD were used to calculate the third-order tensor components of the second-order nonlinear-optical properties, respectively, as shown in Table 4, where $\beta_{\mu}$ is the first hyperpolarisability in the dipole moment direction, and $\beta_{0}$ is the total effective value of the first hyperpolarisability (or static first hyperpolarisability).

It can be seen from Table 4 that the variation trend of the calculated results was consistent with that of the hybrid functional M06-2X and the long-range-corrected hybrid functional WB97XD. However, the value calculated by hybrid functional M06-2X was too large. This was mainly due to the fact that hybrid functional M06-2X is smaller than the HF-type exchange functional mixed in the method of corrected hybrid functional WB97XD, which may overestimate the second-order polarisability. However, the long-range-corrected hybrid functional WB97XD can effectively improve the overestimation of second-order polarisability by DFT exchange functionals due to the mixing of $100 \% \mathrm{HF}$ in the asymptotic region ${ }^{\mathbf{2 7 - 3 0}}$. Therefore, we believe that the calculation results by the WB97XD method are more reliable. From the data in Table 4, it can be seen that the value of $\beta_{\mathrm{x}}$ in the second-order nonlinear-optical coefficient component was relatively large, which indicates that the contribution of $\beta_{\mathrm{x}}$ to $\beta_{\mu}$ (or $\beta_{0}$ ) played a major role, and that the charge transfer within the xy plane was the main reason for the second-order nonlinear-optical properties. The second-order

polarisability of molecules a, b, and $\mathbf{c}$ did not change very much, which indicates that the introduction of conjugated carbon-carbon double bonds or carbon-carbon triple bonds had little effect on the second-order nonlinear-optical properties of the molecules based on the structure of molecule a. For b- and c-series molecules, when a benzene ring, furan ring, or thiophene ring was introduced between the benzene ring and cyanocinnamic acid group, the second-order polarisability 
of the molecules increased, among which the second-order polarisability of molecules $\mathbf{b 3}$ and $\mathbf{c 3}$ obtained by introducing a thiophene ring was the highest. The second-order polarisability of molecules $\mathbf{b 3}$ and $\mathbf{c 3}$ was $0.13 \times 10^{5}$ a.u. and $0.14 \times 10^{5}$ a.u., respectively. Therefore, $\mathbf{b 3}$ and $\mathbf{c} 3$ can be designed into good second-order nonlinear optical materials.

We used the two-level model ${ }^{32,33}$ to qualitatively describe the contribution of molecular charge transfer to the second-order NLO properties:

$\beta \propto \Delta \mu_{\text {eg }} f / E^{3}$ eg

where $\Delta \mu_{\mathrm{eg}}$ is the difference between the dipole moment of the excited state and the dipole moment of the ground state, $f$ is the oscillator strength, and $E_{\text {eg }}$ is the transition energy. From formula (5), it can be seen that for molecules with a larger dipole-moment difference between the excited state and ground state, a smaller transition energy and larger oscillator strength will result in a larger second-order NLO coefficient $\beta$. Table $\mathbf{S 2}$ shows the difference between the excited dipole moment and ground dipole moment of $\mathbf{b}$ - and $\mathbf{c}$-series molecules calculated by the M06-2X/6-311++G(d,p) method. According to the data in the table, the difference between the excited dipole moment and the ground dipole moment of molecules increased when heterocyclic rings were introduced into the triarylamine system.

It can be seen from the data in Table 1 that the transition energy $E_{\text {eg }}$ of five molecules $(\mathbf{a}, \mathbf{b}, \mathbf{c}$, b1, and c1) is close to the calculated result of oscillator strength $f$, but compared with the values of parent molecules $\mathbf{b}$ and $\mathbf{c}$, the transition energy $E_{\text {eg }}$ of four molecules $(\mathbf{b 2}, \mathbf{b 3}, \mathbf{c 2}$, and $\mathbf{c 3})$ decreased, and the oscillator strength $f$ increased significantly. Therefore, the introduction of heterocyclic rings into the triarylamine system enhanced the performance of second-order non-linear optical properties.

\subsubsection{Third-order nonlinear-optical properties}

Using the 6-311++G(d,p) basis set and the finite field (FF) and M06-2X method, an electric field in the range of 0.0010-0.004 a.u was applied in the $\mathrm{x}, \mathrm{y}$, and $\mathrm{z}$ directions, and the charge amplitude was set to 0.00025-0.0005 a.u, as shown in Table 3. The first hyperpolarisability $\beta 0$ of each molecule under different applied electric fields was calculated, respectively, and the calculated results were compared with the calculated total effective $\beta_{0}$ value of the first hyperpolarisability (or the static first hyperpolarisability $\beta_{0}$ ) in the absence of applied electric fields. The electric field with the closest values between the two is the optimal value of applied electric field. The non-zero components and average values of the third-order polarisability of each model molecule of 
triarylamine calculated under applied electric fields are shown in Table 4.

It can be seen from the table that the third-order polarisability non-zero component $\gamma_{\mathrm{xxxx}}$ of the nine molecules is the largest, followed by $\gamma_{y y y y}$, and $\gamma_{z z z z}$ is the smallest, which indicates that the third-order nonlinear-optical properties of triarylamine-derivative molecules was mainly from the charge transfer in the $\mathrm{x}$-axis direction, and the charge transfer in the $\mathrm{y}$-axis direction also contributed to the third-order nonlinear-optical properties. The introduction of carbon-carbon double bonds between biphenyls in molecule a significantly increased the third-order nonlinear-optical properties of the molecules compared to the values for those with carbon-carbon triple bonds. For b- and c-series molecules, after a benzene ring, furan ring or thiophene ring was introduced between the triarylamine and cyanocinnamic acid group, the third-order polarisability increased. The third-order polarisability of $\mathbf{b 2}, \mathbf{b 3}$, and $\mathbf{c 2}, \mathbf{c 3}$ molecules obtained by introducing a furan ring or thiophene ring was relatively large, especially the third-order polarisability of $\mathbf{b 3}$ and $\mathbf{c 3}$ molecules obtained by introducing a thiophene ring, which were the largest at $27.13 \times 10^{5}$ a.u. and $28.10 \times 10^{5}$ a.u., respectively. This shows that molecules with an A- $\pi-\mathrm{D}-\pi-\mathrm{A}$ basic structural unit formed by introducing heteroatomic rings into triarylamine molecules can be designed as good third-order nonlinear-optical materials. 
Table 3. The dipole moment $\mu$ (in a.u.) and second-order non-linear optical properties $\beta$ of nine triarylamine- $\alpha$-cyanoacrylic acid compounds (in $10^{3}$ a.u.).

\begin{tabular}{|c|c|c|c|c|c|c|c|c|c|c|}
\hline Methods & Comp. & $\square \mu_{\mathrm{x}}$ & $\square \mu_{\mathrm{y}}$ & $\square \mu_{\mathrm{z}}$ & $\square \mu$ & $\square \beta_{\mathrm{x}}$ & $\square \beta_{\mathrm{y}}$ & $\square \beta_{\mathrm{z}}$ & $\square \beta_{0}$ & $\square \beta_{\mu}$ \\
\hline \multirow{9}{*}{$\begin{array}{c}\text { M06-2X/ } \\
\text { 6-311++ } \\
\text { G(d,p) }\end{array}$} & $\mathrm{a}$ & -2.8441 & -1.0315 & -0.8519 & 3.1429 & 9.8922 & 3.0788 & -0.0099 & 10.3603 & -7.9381 \\
\hline & $\mathrm{b}$ & 2.1981 & -1.7647 & -0.9604 & 2.9672 & -1.9672 & 10.322 & -0.0321 & 10.5078 & -7.5583 \\
\hline & $\mathrm{c}$ & 2.0841 & -2.3314 & 1.076 & 3.3071 & 1.2831 & 11.3881 & -0.0212 & 11.4601 & -8.8437 \\
\hline & b1 & -1.703 & -0.4688 & -1.9584 & 2.6374 & 13.6901 & 0.2289 & 0.2267 & 10.0731 & -8.5672 \\
\hline & $\mathrm{b} 2$ & -2.833 & 1.3358 & 0.3516 & 3.1518 & 15.7162 & -2.3258 & 1.3949 & 15.9486 & -14.9566 \\
\hline & b3 & 2.5923 & 1.5606 & 0.2125 & 3.0333 & 17.0681 & 0.7452 & 0.2227 & 17.0857 & -14.9546 \\
\hline & $\mathrm{c} 1$ & -1.907 & -0.4981 & -1.9424 & 2.7675 & 13.9657 & -0.3629 & 0.2118 & 13.9721 & -9.7084 \\
\hline & $\mathrm{c} 2$ & -2.647 & 2.0092 & 0.3583 & 3.3422 & 15.3891 & -5.9627 & 1.4083 & 16.5639 & -15.6203 \\
\hline & $\mathrm{c} 3$ & 2.6215 & 1.8616 & -0.1978 & 3.2214 & -17.696 & -2.4282 & -0.1401 & 17.8621 & -15.7954 \\
\hline \multirow{9}{*}{$\begin{array}{c}\text { WB97Xd } \\
\text { /6-311++ } \\
\text { G(d,p) }\end{array}$} & $\mathrm{a}$ & -2.8274 & -1.0787 & -0.8708 & 3.1429 & 8.589 & 2.699 & -0.0213 & 9.0031 & -6.7816 \\
\hline & $\mathrm{b}$ & 2.2306 & -1.7305 & -0.9814 & 2.9888 & -1.6561 & 8.7264 & -0.0437 & 8.8822 & -6.2739 \\
\hline & $\mathrm{c}$ & 2.1289 & -2.0291 & -0.9757 & 3.0986 & -0.8785 & 8.4212 & -0.0035 & 8.4669 & -6.1991 \\
\hline & b1 & -1.6850 & -0.4797 & -2.0002 & 2.6589 & 9.2814 & 0.1691 & 0.1606 & 9.2843 & -5.9721 \\
\hline & b2 & -2.8393 & 1.3693 & 0.3714 & 3.1741 & 12.5239 & -1.5945 & 1.2768 & 12.6894 & -11.7415 \\
\hline & b3 & -2.5855 & 1.5889 & 0.2175 & 3.0425 & 13.1481 & 0.3399 & 0.2053 & 13.1541 & -11.3359 \\
\hline & $\mathrm{c} 1$ & -1.8876 & -0.5098 & -1.9840 & 2.7855 & 9.8064 & -0.2589 & 0.1479 & 9.8109 & -6.7032 \\
\hline & $\mathrm{c} 2$ & -2.6400 & 2.0228 & 0.3848 & 3.3481 & 12.2556 & -4.3897 & 1.2804 & 13.0808 & -12.1688 \\
\hline & c3 & 2.6099 & 1.8890 & -0.2026 & 3.2281 & -13.5662 & -1.6596 & -0.1364 & 13.6681 & -11.9307 \\
\hline
\end{tabular}


Table 4. The third-order nonlinear-optical coefficients $\gamma$ of nine triarylamine- $\alpha$-cyanoacrylic acid compounds at the M06-2X/6-311++G(d,p) theoretical level (in $10^{5}$ a.u.).

\begin{tabular}{cccccccc}
\hline Comp. & $\square \gamma_{\text {xxx }}$ & $\square \gamma_{\text {xxyy }}$ & $\square \gamma_{\text {xxzz }}$ & $\square \gamma_{\text {yyyy }}$ & $\square \gamma_{\text {yyzz }}$ & $\square \gamma_{\text {zzzz }}$ & $\square \gamma$ \\
\hline $\mathbf{a}$ & 19.75 & 3.37 & 0.37 & 7.25 & 0.25 & 1.01 & 6.90 \\
$\mathbf{b}$ & 25.68 & 7.91 & 0.00 & 25.68 & -0.59 & 1.97 & 13.59 \\
$\mathbf{c}$ & 37.53 & 13.83 & 5.93 & 33.58 & 5.93 & 5.93 & 25.67 \\
$\mathbf{b 1}$ & 72.49 & 10.27 & 0.00 & 31.21 & 0.00 & 0.59 & 24.97 \\
$\mathbf{b 2}$ & 78.13 & 11.88 & 0.63 & 29.38 & -1.25 & -0.63 & 25.88 \\
$\mathbf{b 3}$ & 86.88 & 11.25 & -0.63 & 28.75 & -1.25 & 1.25 & 27.13 \\
$\mathbf{c 1}$ & 80.00 & 20.00 & 0.00 & 30.00 & 0.00 & -19.99 & 26.00 \\
$\mathbf{c 2}$ & 69.12 & 16.62 & 0.49 & 35.19 & 0.13 & 0.49 & 27.94 \\
$\mathbf{c 3}$ & 49.99 & 10.00 & -10.00 & 59.99 & 9.99 & 10.00 & 28.00 \\
\hline
\end{tabular}

\section{Conclusions}

We used the hybrid DFT M06-2X method with the 6-311++g(d,p) basis set to optimise the structures, calculate the frequencies, analyse the absorption spectra, and evaluate the NPA charges of nine triarylamine derivatives. Nonlinear-optical properties were computed with the hybrid DFT M06-2X, long-range-corrected hybrid functional WB97XD, and FF methods. The following conclusions were reached:

(1) The lowest energy transition of the nine triarylamine derivatives molecules was the $\pi-\pi^{*}$ electron transition from HOMO to LUMO. Compared with those of the gas phase, the maximum absorption wavelengths of the molecules in ethanol solution exhibited a certain degree of redshift (about 6-31 nm).

(2) The long-range-corrected hybrid functional WB97XD method was more reliable for predicting the second-order nonlinear-optical properties of the molecules.

(3) Based on parent molecule a, the introduction of conjugated carbon-carbon double bonds and carbon-carbon triple bonds has little effect on the second-order nonlinear-optical properties of the molecules. However, the introduction of carbon-carbon double bonds into the parent molecules improves the third-order nonlinear-optical properties. The introduction of a heterocyclic ring into the triarylamine system enhances the second-order and third-order nonlinear-optical properties, especially the second-order and third-order polarisability of the b3 and $\mathbf{c} 3$ molecules obtained by introducing a thiophene ring; these molecules had the highest polarisability values. This suggests that the $\mathbf{b 3}$ and $\mathbf{c 3}$ molecules have beneficial second- and third-order nonlinear-optical 
properties, and can be used as the basis for the design of good second- and third-order nonlinear-optical materials.

\section{Declarations}

\section{Acknowledgements}

We would like to thank Editage (www.editage.cn) for English language editing. And we are grateful to the reviewers for their invaluable suggestions.

\section{Funding}

This work was supported by the Project of Science and Technology Department of Sichuan Province (Number 2015GZ0343 of prof. Ziran Chen), the Natural Science Foundation of Sichuan Vocational and Technical College (Number 2022YZA003 of prof. Ziran Chen; 2022YZB005 of Dr. Yuan Li).

Conflicts of interest/Competing interests The authors declare no competing interests.

Data availability Not applicable.

Code availability NA

Author contribution Ziran Chen: methods, writing, and data analysis.

Yuhong Zhang: result analysis, manuscript first draft.

Yuan Li: simulations, analysis, manuscript editing.

Wenhao Yu: project management, manuscript editing.

\section{REFERENCES}

[1] Rajeshirke M, Sekar N 2019 Multi-stimuli responsive emissive NLOphoric colorants - A recent trend in research Dyes Pigments 163675.

[2] Marder S-R 2006 Organic nonlinear optical materials: where we have been and where we are going Chem. Comm. 131

[3] Liu X-H, Zhao Z-X, Wang J, Zhang W and Zhang H-X. 2017 Theoretical investigation on the photoswitchable second-order nonlinear optical properties of a series of $\mathrm{B}(\mathrm{C} 6 \mathrm{~F} 5) 2$-coordinated dithienylethene derivatives. J. Photoch. Photobio. A: Chem.335 155

[4] Chen Z, Li Y, Guan Y and Li H. 2019 Rational design of the nonlinear optical materials dinaphtho [2,3-b:2', 3'-d]thiophene-5,7,12,13-tetraone (DNTTRA) and its phenyldiazenyl derivatives using first-principles calculations J. Comput. Electron. 186 
[5] CHEN Z-R, LI Q and Zhao K-Q 2011 Electronic spectrum and the secord-order nonlinear optical properties of pyrazoline- oxadiazole organic molecules Acta Chim. Sinica 692908

[6] Chen J, Wang M-Q, Zhou X, Yang L, Li W-Q and Tian W-Q 2017 Two-dimensional second-order nonlinear optical spectra: landscape of second-order nonlinear optics Phys. Chem. Chem. Phys. 1929315

[7] Qi D and Jiang J 2015 Nature of the Intense Second-Order Nonlinear Optical Activity: DFT Studies on the Octupolarization of Sandwich-Type Bis(phthalocyaninato) Yttrium Skeletons ChemPhysChem 161889

[8] Kim T-D, Kang J-W, Luo J, Jang S-H, Ka J-W, Tucker N, Benedict JB, Dalton LR, Gray T, Overney RM, Park DH, Herman WN and Jen AKY 2007 Ultralarge and Thermally Stable Electro-Optic Activities from Supramolecular Self-Assembled Molecular Glasses. J. Am. Chem. Soc. 129488

[9] Wu J-B, Wilson B-A, Smith D-W and Nielsen S-O 2014 Towards an understanding of structure-nonlinearity relationships in triarylamine-based push-pull electro-optic chromophores: the influence of substituent and molecular conformation on molecular hyperpolarizabilities J. Mater. Chem. C 22591

[10] Misra R 2017 Tuning of Second-Order Nonlinear Optical Response Properties of Aryl-Substituted Boron-Dipyrromethene Dyes: Unidirectional Charge Transfer Coupled with Structural Tailoring J. Phys.Chem. C 1215731

[11] Shkir M. 2017 Investigation on the key features of L-Histidinium 2-nitrobenzoate (LH2NB) for optoelectronic applications: A comparative study J. King Saud Univ. Sci. 2970

[12] Shkir M, Patil P-S, Arora M, AlFaify S and Algarni H 2017 An experimental and theoretical study on a novel donor- $\pi$-acceptor bridge type 2, 4, 5-trimethoxy-4'-chlorochalcone for optoelectronic applications: A dual approach Spectrochim. Acta A: Mol. Biomol. Spectrosc. 173 445

[13] Shkir M, AlFaify S, Arora M, Ganesh V, Abbas H and Yahia I-S 2018 A first principles study of key electronic, optical, second and third order nonlinear optical properties of 3-(4-chlorophenyl)-1-(pyridin-3-yl) prop-2-en-1-one: a novel D- $\pi$-A type chalcone derivative $J$. Comput. Electron. 179

[14] AlFaify S, Shkir M, Arora M, Irfan A, Algarni H, Abbas H and Al-Sehemi AG 2018 Quantum 
chemical investigation on molecular structure, vibrational, photophysical and nonlinear optical properties of 1-threoninium picrate: an admirable contender for nonlinear applications. $J$. Comput. Electron.17 1421

[15] Wazzan N. 2020 The nonlinear optical properties of acyclic triarylamine-conjugated dimethyl diethynylfumarate and its two cyclic Pechmann dyes derivatives: A theoretical study Optik 207

[16] Chen Z-R, Zhang Y, He Z-R, Guan Y, Li Y and Li H 2020 Electronic Absorption Spectra and Third-Order Nonlinear Optical Property of Dinaphtho[2,3-b:2',3'-d]Thiophene-5,7,12,13Tetraone (DNTTRA) and Its Phenyldiazenyl Derivatives: DFT Calculations. Comput. Chem. 8 43

[17] Singer KD, Sohn JE, King LA, Gordon H-M, Katz H-E and Dirk C-WSecond-order nonlinear-optical properties of donor- and acceptor-substituted aromatic compounds J. Opt. Soc. Am. B 1989;6 1339

[18] Wielopolski M, Kim J-H, Jung Y-S, Yu Y-J, Kay K-Y, Holcombe TW, Zakeeruddin SM, Grätzel M and Moser J-E 2013 Position-Dependent Extension of $\pi$-Conjugation in D- $\pi$-A Dye Sensitizers and the Impact on the Charge-Transfer Properties J. Phys. Chem. C 11713805

[19] Qian X, Chang W-Y, Zhu Y-Z, Wang S-S, Pan B, Lu L and Zheng JY 2015 Tri(N-carbazolyl)triphenylamine-based starburst organic dyes: effects of different acceptors on the optical, electrochemical and photovoltaic properties RSC Adv. 547422

[20] Fahim ZME, Bouzzine SM, Hamidi MM, Bouachrine M, Hamidi M, Salgado-Moran G, Mendoza-Huizar LH and Alvarez-Romero GA 2018 Optoelectronic properties of triophenylamine based dyes for solar cell applications. A DFT study Quim. Nova 41129

[21] Janjua M, Khan MU, Khalid M, Ullah N, Kalgaonkar R, Alnoaimi K, Baqader N and Jamil S 2021 Theoretical and Conceptual Framework to Design Efficient Dye-Sensitized Solar Cells (DSSCs): Molecular Engineering by DFT Method J. Clust. Sci. 32243

[22] Janjua MRSA, Khan M-U, Bashir B, Iqbal M-A, Song Y, Naqvi S-A-R and Khan Z-A 2012 Effect of $\pi$-conjugation spacer (CC) on the first hyperpolarizabilities of polymeric chain containing polyoxometalate cluster as a side-chain pendant: A DFT study Comput. Theor. Chem. 99434

[23] Vineetha PK, Aswathy A, Shiju E, Chandrasekharan K and Manoj N 2020 Structure-property correlations of the nonlinear optical properties of a few bipodal D-pi-A molecules - an 
experimental and theoretical approach New J. Chem. 446142

[24] Janjua M 2018 Density Functional Theory Predictions of the Nonlinear Optical (NLO) Properties in Triphenylamine based alpha-Cyanocinnamic Acid Compounds: Effect of Fluorine on NLO Response J. Mex. Chem. Soc. 62125

[25] M. J. Frisch, G. W. Trucks, H. B. Schlegel, G. E. Scuseria, M. A. Robb, J. R. Cheeseman, G. Scalmani, V. Barone, G. A. Petersson, H. Nakatsuji, X. Li, M. Caricato, A. V. Marenich, J. Bloino, B. G. Janesko, R. Gomperts, B. Mennucci, H. P. Hratchian, J. V. Ortiz, A. F. Izmaylov, J. L. Sonnenberg, D. Williams-Young, F. Ding, F. Lipparini, F. Egidi, J. Goings, B. Peng, A. Petrone, T. Henderson, D. Ranasinghe, V. G. Zakrzewski, J. Gao, N. Rega, G. Zheng, W. Liang, M. Hada, M. Ehara, K. Toyota, R. Fukuda, J. Hasegawa, M. Ishida, T. Nakajima, Y. Honda, O. Kitao, H. Nakai, T. Vreven, K. Throssell, J. A. Montgomery, Jr., J. E. P., F. Ogliaro, M. J. Bearpark, J. J. Heyd, E. N. Brothers, K. N. Kudin, V. N. Staroverov, T. A. Keith, R. Kobayashi, J. Normand, K. Raghavachari, A. P. Rendell, J. C. Burant, S. S. Iyengar, J. Tomasi, M. Cossi, J. M. Millam, M. Klene, C. Adamo, R. Cammi, J. W. Ochterski, R. L. Martin, K. Morokuma, O. Farkas, J. B. Foresman and Fox, D. J 2016 Gaussian 16, Gaussian, Inc., Wallingford CT

[26] Hristova S, Deneva V, Pittelkow M, Crochet A, Kamounah FS, Fromm KM, Hansen PE and Antonov L 2018 A concept for stimulated proton transfer in 1-(phenyldiazenyl)naphthalen-2-ols Dyes Pigments 15691

[27] Jacquemin D, Perpète EA, Medved' M, Scalmani G, Frisch MJ, Kobayashi R and Adamo C 2007 First hyperpolarizability of polymethineimine with long-range corrected functionals. $J$. Chem. Phys. 126:191108

[28] Sekino H, Maeda Y, Kamiya M and Hirao K 2007 Polarizability and second hyperpolarizability evaluation of long molecules by the density functional theory with long-range correction $J$. Chem. Phys. 126014107

[29] Chai J-D and Head-Gordon M 2008 Long-range corrected hybrid density functionals with damped atom-atom dispersion corrections Phys. Chem. Chem. Phys. 106615

[30] Jian L and Zhang M-X 2016 Theoretical study on the second-order nonlinear optical properties andswitching properties of dithienylethene containing boron molecules J. Atom. Mol. Phys. 3313 
[31] Wang H, Bao B, Hu X and Fang J-K 2017 Efficient Small Molecule Organic Dyes Containing Different Bridges in Donor Moieties for Dye-Sensitized Solar Cells Electrochim. Acta 250278

[32] Oudar J-L 1977 Optical nonlinearities of conjugated molecules. Stilbene derivatives and highly polar aromatic compounds J. Chem. Phys.67 446

[33] Mallah R-R, Mohbiya D-R, Sreenath M-C, Chitrambalam S, Joe I-H and Sekar N 2019 Non-linear optical response of meso hybrid BODIPY: Synthesis, photophysical, DFT and Z scan study Spectrochim. Acta A: Mol. Biomol. Spectrosc. 209126 


\section{Figures}

\section{Figure 1}

The chemical structures of nine triarylamine-a-cyanoacrylic acid compounds.

\section{Figure 2}

HOMO energies $\left(E_{H}\right)$, LUMO energies $\left(E_{\mathrm{L}}\right)$, and HOMO-LUMO gaps $\left(E_{g}\right)$ of nine triarylamine-a-cyanoacrylic acid compounds.

\section{Supplementary Files}

This is a list of supplementary files associated with this preprint. Click to download.

- GraphicalAbstracts.png

- 2021NLOSI.doc 